Sains Malaysiana 49(2)(2020): 315-322

http://dx.doi.org/10.17576/jsm-2020-4902-09

\title{
Synthesis of Cinnamamide Derivatives and Their $\alpha$-Glucosidase Inhibitory Activities
}

(Sintesis Terbitan Sinamamida dan Aktiviti Rencatan $\alpha$-Glucosidase)

\author{
Teni ERnawati*, Abdul Mun'Im, Muhamad Hanafi \& ARry Yanuar
}

\begin{abstract}
Chemically, methyl trans-cinnamate offers treatment at the three main reactive sites such as substitution at the phenyl ring, addition at the $\alpha, \beta$-unsaturation, and substitution at the carboxylic methyl ester functionality. We focus our research to the amide and related derivatives of cinnamates because of their lesser known attributes towards antidiabetic activities. In this research, we modify methyl trans-cinnamate by amidating the carboxylic methyl ester functionality using several amines introduced in functional groups of methyl trans-cinnamate. A series of cinnamamide derivatives was synthesized and evaluated for $\alpha$-glucosidase inhibitory effects. The structure of synthesized compounds was characterized by IR, melting point, UV, ${ }^{1} \mathrm{H} N M R,{ }^{13} \mathrm{C} N M R$, and mass spectral analysis. All 13 cinnamamide showed higher $\alpha$-glucosidase activity than the starting compound. The substitution of cinnamic acid with an amide group altered the a-glucosidase inhibitory activity. Increased bulkiness and the chain length of the amine substituents decreased the inhibitory activity. Propylcinnamamide (3c) showed the most potent inhibitory activity among all the cinnamamide derivatives, all of which act through a competitive inhibitory mechanism. These compounds may be worth exploring further.
\end{abstract}

Keywords: $\alpha$-glucosidase inhibitor; cinnamamide derivative; synthesis

ABSTRAK

Secara kimia, metil trans-sinamat menawarkan rawatan pada tiga tapak reaktif utama seperti penggantian pada gelang fenil, penambahan pada ketaktepuan $\alpha, \beta$ dan penggantian pada kefungsian karbosilik metil ester. Kami memfokuskan kajian ini kepada amida dan terbitan berkaitan sinamat kerana atribut yang kurang dikenali terhadap aktiviti antidiabetis. Dalam kajian ini kami mengubah suai metil trans-sinamat dengan keamidaan kefungsian karbosilik metil ester menggunakan beberapa amina yang diperkenalkan dalam kumpulan fungsian metil trans-sinamat. Beberapa terbitan sinamamida telah disintesis dan dinilai untuk kesan rencatan $\alpha$-glucosidase. Struktur sintesis sebatian telah dicirikan oleh IR, titik lebur, UV, ${ }^{1} \mathrm{H} N M R,{ }^{13} \mathrm{C} N M R$ dan analisis jisim spektrum. Kesemua 13 sinamamida menunjukkan aktiviti $\alpha$-glucosidase yang tinggi berbanding sebatian pemula. Penggantian asid sinamik dengan kumpulan amida mengubah aktiviti perencatan a-glucosidase. Peningkatan berpukal dan panjang rantai bahan ganti amina mengurangkan aktiviti rencatan. Propilsinamamida (3c) menunjukkan aktiviti rencatan poten dalam kalangan terbitan sinamamida, dengan kesemuanya bertindak melalui mekanisme perencatan berpersaingan. Sebatian ini mungkin berbaloi untuk kajian lanjut.

Kata kunci: Perencat $\alpha$-glucosidase; sintesis; terbitan sinamamida

\section{INTRODUCTION}

$\alpha$-Glucosidase is a type of hydrolase enzyme that catalyzes the hydrolysis of terminal non-reducing carbohydrates into $\alpha$-glucose (Park et al. 2013). Inhibition of $\alpha$-glucosidase enzyme activity allows blood glucose levels to return within normal range (Bösenberg \& van Zyl 2008). Glucosidase is responsible for the catalytic disruption of glycosidic bonds, which specifically depends on the amount of monosaccharides, the position of the cleavage site, and the configuration of the hydroxyl group in the substrate (Park et al. 2008a). Enzymatic hydrolysis of glycosidic bonds occurs through acid catalysis that requires two important residues such as proton donors and bases/ nucleophiles (Park et al. 2008b). $\alpha$-Glucosidase inhibitors
(IAGs), such as acarbose, nojirimycin, 1-deoxynojirimycin, miglitol, and voglibose, are widely used for the treatment of type 2 diabetes and obesity (Bösenberg \& van Zyl 2008; Davies \& Henrissat 1995; Liu et al. 2006; Mohammed et al. 2014; Van de Laar et al. 2008). They can also be used as a therapeutic target to treat viral infections, cancers, and hepatitis (Fischer et al. 1995; Mehta et al. 1998; Melo \& Carvalho 2006).

Cinnamic acid derivatives have been reported to inhibit $\alpha$-glucosidase (Babu et al. 2007; Huang et al. 2009; Narender et al. 2007; Phuwapraisirisan et al. 2008; Pili et al. 1995). The pharmacophore of cinnamic acid compound are phenyl ring, $\alpha, \beta$-unsaturation and carboxylic acid. It is known that cinnamic acid derivatives act as IAGs. Their 
structure includes a substitution at para position within the aromatic functional group functionalized with an alkylester group (Matsuura et al. 2004). However, no cinnamamide derivatives have been tested for their $\alpha$-glucosidase inhibitory ability. Therefore, we replaced the carboxylic acid moiety of cinnamic acid by an alkyl amine and predicted that it can have better inhibitory activity against $\alpha$-glucosidase. In this study, we synthesized a series of cinnamamide derivatives and elucidated their structures with H-NMR, C-NMR, FTIR, ESI QToF MS, GCMS. We report their inhibitory effects against Saccharomyces cerevisiae and rat intestinal $\alpha$-glucosidase.

\section{MATERIALS AND METHODS}

\section{CHEMICAL AND INSTRUMENTS}

All solvents were dried and distilled according to standard procedure. ${ }^{1} \mathrm{H}$ were recorded on JEOL NMR ECZ500R using $500 \mathrm{MHz}$ instrument and ${ }^{13} \mathrm{C}$ NMR spectra were recorded on JEOL NMR ECZ500R using $125 \mathrm{MHz}$ instrument in deuterochloroform. Chemical shifts $(\delta)$ are reported in parts per million (ppm) downfield from tetramethylsilane $(\delta 0.00)$ or $\mathrm{CHCl}_{3}(\delta 7.26)$ for ${ }^{1} \mathrm{H} \mathrm{NMR}$ and $\delta 77.0$ for ${ }^{13} \mathrm{C}$ NMR as an internal standard, and coupling constants are reported in Hertz. ESI QToF MS Mass Spectra were recorded on Waters Xevo ${ }^{\circledR}$ G2-XS QToF. IR spectra were obtained on Bruker Tensor II. Analytical thin layer chromatography (TLC) was performed on Merck silica gel plates (Art5715 Kiesel gel $60 \mathrm{~F}_{254} 0.25 \mathrm{~mm}$ ) and preparative TLC was performed using Merck silica gel plates (Art5744 Kiesel gel $60 \mathrm{~F}_{254} 0.5 \mathrm{~mm}$ ). Silica gel column chromatography was carried out on Daisogel IR-60. Methyl transcinnamates obtained from isolation of Alpinia malaccensis using hydrodistillation methode was chosen as the starting material, which was characterized by GCMS, LCMS, ESIQToF MS, ${ }^{1} \mathrm{H}$ NMR, ${ }^{13} \mathrm{C}$ NMR, and FTIR.

\section{EXPERIMENTS}

Hydrolysis of methyl trans-cinnamate

In a $250 \mathrm{~mL}$ round-bottomed flask, methyl trans-cinnamate $(0.012 \mathrm{~mol}), 2 \mathrm{M} \mathrm{NaOH}$, and $95 \%$ ethanol were added. The reaction mixture was heated at $50-60^{\circ} \mathrm{C}$ for $3 \mathrm{~h}$. After completion (monitored by TLC), the reaction was neutralized with $\mathrm{HCl} 1 \mathrm{M}$; the reaction mixture was extracted with butanol. The organic phase was washed with water, dried over anhydrous $\mathrm{Na}_{2} \mathrm{SO}_{4}$, and concentrated under reduced pressure to afford the product. Cinnamic acid was obtained by recrystallization. Identification of cinnamic acid was performed by using FT-IR, LC-MS, ${ }^{1} \mathrm{H}-\mathrm{NMR}$, and ${ }^{13} \mathrm{C}-\mathrm{NMR}$ and GC-MS.

Cinnamic acid (2). Yield: (1.51 g, 85\%). Mp: 133$134^{\circ} \mathrm{C}$. ESI-QToF MS $m / z 149.09745[\mathrm{M}+\mathrm{H}]^{+}$. FTIR: $v_{\mathrm{ma}}$ $\left(\mathrm{cm}^{-1}\right), 3680(\mathrm{C}-\mathrm{OH}), 1685(\mathrm{C}=\mathrm{O}) .{ }^{1} \mathrm{H}-\mathrm{NMR}(500 \mathrm{MHz}$, $\left.\mathrm{CDCl}_{3}\right) \delta 6.49(d, 1 \mathrm{H}, J=16 \mathrm{~Hz}), 7.83(d, 1 \mathrm{H}, J=16 \mathrm{~Hz})$, $7.57(d d, 2 \mathrm{H}, J=2.0, \mathrm{~Hz} ; J=7.8 \mathrm{~Hz}), 7.42(d t, 3 \mathrm{H}, J=$ $2.0, J=7.2 \mathrm{~Hz}),{ }^{13} \mathrm{C}-\mathrm{NMR}\left(125 \mathrm{MHz}, \mathrm{CDCl}_{3}\right) \delta 172.8,117.4$, $147.2,134.1,128.5,129.1,130.8,129.1,128.5$.
Amidation of cinnamic acid compound (series 3)

In a $250 \mathrm{~mL}$ flask, $\mathrm{SOCl}_{2}(4 \mathrm{~mL}, 0.055 \mathrm{~mol})$ was added dropwise to cinnamic acid $(0.2961 \mathrm{~g}, 2 \mathrm{mmol})$ at $0^{\circ} \mathrm{C}$ and then heated for $5 \mathrm{~h}$. The reaction mixture was cooled and anh. $\mathrm{CH}_{2} \mathrm{Cl}_{2}(10 \mathrm{~mL})$, pyridine $0.2 \mathrm{~mL}$, and (amine, ethylamine, propylamine, isopropylamine, octylamine, phenylamine, $p$-methoxyphenylamine) $(6 \mathrm{mmol})$ were added and then stirred at room temperature for $3 \mathrm{~h}$. The reaction mixture was washed with saturated $\mathrm{NaCl}$ and extracted with $\mathrm{CH}_{2} \mathrm{Cl}_{2}$. The organic layer was dried to give a crude product, which was purified by column chromatography (hex:AcOEt: 4:1) (Adisakwattana et al. 2004; Carvalho et al. 2008).

\section{Cinnamamide (3a)}

Yield: (0.235 g, 80\%). Mp: $145-148^{\circ} \mathrm{C}$. ESI-QToF MS $m / z$ $148.07485[\mathrm{M}+\mathrm{H}]^{+}$. FTIR: $v_{\max }\left(\mathrm{cm}^{-1}\right), 3369(\mathrm{NH}), 1662$ $(\mathrm{C}=\mathrm{O}) .{ }^{1} \mathrm{H}-\mathrm{NMR}\left(500 \mathrm{MHz}, \mathrm{CDCl}_{3}\right) \delta 6.43(d, 1 \mathrm{H}, J=16$ $\mathrm{Hz}), 7.64(d, 1 \mathrm{H}, J=16 \mathrm{~Hz}), 7.47$ (dd, 2H, $J=9.75 \mathrm{~Hz})$, $7.35(d t, 3 \mathrm{H}, J=3.9 \mathrm{~Hz}, J=9.75 \mathrm{~Hz}),{ }^{13} \mathrm{C}-\mathrm{NMR}(125 \mathrm{MHz}$, $\left.\mathrm{CDCl}_{3}\right) \delta 168.3,119.2,143.1,134.5,128.1,128.9,130.2$, 128.9, 128.1 .

\section{Ethylcinnamamide (3b)}

Yield: (0.262 g, 75\%). Mp: $85-87^{\circ} \mathrm{C}$. LCMS m/z 176.39 $[\mathrm{M}+\mathrm{H}]^{+}$, FTIR: $v_{\max }\left(\mathrm{cm}^{-1}\right), 3284(\mathrm{NH}), 1656(\mathrm{C}=\mathrm{O})$. ${ }^{1} \mathrm{H}-\mathrm{NMR}\left(500 \mathrm{MHz}, \mathrm{CDCl}_{3}\right) \delta 6.43(d, 1 \mathrm{H}, J=16 \mathrm{~Hz}), 7.64$ $(d, 1 \mathrm{H}, J=16 \mathrm{~Hz}), 7.47(d d, 2 \mathrm{H}, J=7.8 \mathrm{~Hz}), 7.35(t, 3 \mathrm{H}$, $J=1.95 \mathrm{~Hz}, J=7.8 \mathrm{~Hz}), 3.42(\mathrm{q}, 2 \mathrm{H}, J=2.6 \mathrm{~Hz}), 1.20(t$, $1 \mathrm{H}, J=7.15 \mathrm{~Hz}),{ }^{13} \mathrm{C}-\mathrm{NMR}\left(125 \mathrm{MHz}, \mathrm{CDCl}_{3}\right) \delta 166.4$, $121.4,140.5,135.0,127.8,128.8,129.6,128.8,127.8$, $34.7,14.8$.

\section{Propylcinnamamide (3c)}

Yield: $(0.31 \mathrm{~g}, 82 \%)$. Mp: $74-75^{\circ} \mathrm{C}$. ESI-QToF MS $\mathrm{m} / \mathrm{z}$ $190.15952[\mathrm{M}+\mathrm{H}]^{+}$. FTIR: $v_{\max }\left(\mathrm{cm}^{-1}\right), 3269(\mathrm{NH}), 1654$ $(\mathrm{C}=\mathrm{O}) .{ }^{1} \mathrm{H}-\mathrm{NMR}\left(500 \mathrm{MHz}, \mathrm{CDCl}_{3}\right) \delta 6.48(d, 1 \mathrm{H}, J=16$ $\mathrm{Hz}), 7.60(d, 1 \mathrm{H}, J=16 \mathrm{~Hz}), 7.45(d d, 2 \mathrm{H}, J=7.8 \mathrm{~Hz})$, $7.30(d t, 3 \mathrm{H}, J=3.25 \mathrm{~Hz}, J=7.8 \mathrm{~Hz}), 3.34(q, 2 \mathrm{H}, J=$ $6.5 \mathrm{~Hz}), 1.58(m, 2 \mathrm{H}, J=7.15 \mathrm{~Hz}), 0.93(t, 3 \mathrm{H}, J=7.75$ $\mathrm{Hz}),{ }^{13} \mathrm{C}-\mathrm{NMR}\left(125 \mathrm{MHz}, \mathrm{CDCl}_{3}\right) \delta 166.4,121.2,140.7$, $135.0,127.8,128.8,129.6,128.8,127.8,41.6,22.9,11.6$.

\section{Isopropylcinnamamide (3d)}

Yield: (0.295 g, $78 \%$ ). Mp: $95-97^{\circ} \mathrm{C}$. ESI-QToF MS $\mathrm{m} / \mathrm{z}$ $190.1568[\mathrm{M}+\mathrm{H}]^{+}$. FTIR: $v_{\max }\left(\mathrm{cm}^{-1}\right), 3265(\mathrm{NH}), 1656$ $(\mathrm{C}=\mathrm{O}) .{ }^{1} \mathrm{H}-\mathrm{NMR}\left(500 \mathrm{MHz}, \mathrm{CDCl}_{3}\right) \delta 6.43(d, 1 \mathrm{H}, J=16$ $\mathrm{Hz}), 7.63(d, 1 \mathrm{H}, J=16 \mathrm{~Hz}), 7.47(d d, 2 \mathrm{H}, J=7.8 \mathrm{~Hz})$, $7.32(t, 3 \mathrm{H}, J=4.5 \mathrm{~Hz}, J=7.8 \mathrm{~Hz}), 4.22(m, 1 \mathrm{H}, J=7.15$ $\mathrm{Hz}), 1.22(d, 3 \mathrm{H}, J=7.1 \mathrm{~Hz}), 1.22(d, 3 \mathrm{H}, J=7.1 \mathrm{~Hz}),{ }^{13} \mathrm{C}-$ NMR $\left(125 \mathrm{MHz}, \mathrm{CDCl}_{3}\right) \delta 165.3,121.2,140.8,134.9,128.2$, $128.8,129.6,128.8,128.2,41.7,22.9,22.9$.

\section{Octylcinnamamide (3e)}

Yield: $(0.477$ g, $82.4 \%)$. Mp: $74-75^{\circ} \mathrm{C}$. ESI-QToF MS $m / z$ 259.23412 [M+H] . FTIR: $v_{\max }\left(\mathrm{cm}^{-1}\right), 3282(\mathrm{NH}), 1631$ $(\mathrm{C}=\mathrm{O}) 3059$ and 2926 (CH aliphatic). ${ }^{1} \mathrm{H}-\mathrm{NMR}$ (500 MHz, $\mathrm{CDCl}_{3}$ ) 
$\delta 0.85(\mathrm{t}, J=6.5 \mathrm{~Hz}, 3 \mathrm{H}), 1.56(m, 2 \mathrm{H}, J=7.8 \mathrm{~Hz})$, $1.24(m, 2 \mathrm{H}, J=3.9 \mathrm{~Hz}), 1.24(m, 2 \mathrm{H}, J=3.9 \mathrm{~Hz}), 1.24$ $(m, 2 \mathrm{H}, J=3.9 \mathrm{~Hz}), 1.24(m, 2 \mathrm{H}, J=3.9 \mathrm{~Hz}), 1.24(m$, $2 \mathrm{H}, J=3.9 \mathrm{~Hz}), 3.37(q, 2 \mathrm{H}, J=6.5 \mathrm{~Hz}), 7.48(d d, 2 \mathrm{H}, J$ $=7.8 \mathrm{~Hz}), 7.32(d t, 3 \mathrm{H}, J=1.95 \mathrm{~Hz}, J=9.05 \mathrm{~Hz}), 7.64(d$, $1 \mathrm{H}, J=16 \mathrm{~Hz}), 6.46(d, 1 \mathrm{H}, J=16 \mathrm{~Hz}),{ }^{13} \mathrm{C}-\mathrm{NMR}(125$ $\left.\mathrm{MHz}, \mathrm{CDCl}_{3}\right) \delta 14.2,22.7,31.8,29.3,29.4,27.1,29.7,40.0$, $127.9,128.9,129.7,128.9,127.9,134.9,140.9,120.8$, 166.2 .

\section{Phenylcinnamamide (3f)}

Yield: (0.348 g, $78 \%$ ). Mp: $77-79^{\circ} \mathrm{C}$. ESI-QToF MS $m / z$ $224.10618[\mathrm{M}+\mathrm{H}]^{+}$. FTIR: $v_{\max }\left(\mathrm{cm}^{-1}\right), 3271(\mathrm{NH}), 1604$ $(\mathrm{C}=\mathrm{O}) .{ }^{1} \mathrm{H}-\mathrm{NMR}\left(500 \mathrm{MHz}, \mathrm{CDCl}_{3}\right) \delta 6.69(d, 1 \mathrm{H}, J=16$ $\mathrm{Hz}), 7.16(d, 1 \mathrm{H}, J=16 \mathrm{~Hz}), 7.18(d d, 2 \mathrm{H}, J=7.15 \mathrm{~Hz})$, $6.78(d t, 2 \mathrm{H}, J=2.6 \mathrm{~Hz}, J=8.4 \mathrm{~Hz}), 6.76(d t, 1 \mathrm{H}, J=2.6$ $\mathrm{Hz}, J=8.4 \mathrm{~Hz}), 7.18(d d, 2 \mathrm{H}, J=8.4 \mathrm{~Hz}), 6.71(d t, 2 \mathrm{H}$, $J=2.6 \mathrm{~Hz}, J=8.4 \mathrm{~Hz}), 6.69(d t, 1 \mathrm{H}, J=2.6 \mathrm{~Hz}, J=8.4$ $\mathrm{Hz}),{ }^{13} \mathrm{C}-\mathrm{NMR}\left(125 \mathrm{MHz}, \mathrm{CDCl}_{3}\right) \delta 164.7,120.4,142.4$, $134.9,129.1,128.9,124.6,128.9,129.1,138.2,121.2$, $128.1,130.0,128.1,121.2$.

\section{p-Methoxyphenylcinnamamide $(3 g)$}

Yield: (0.394 g, $78 \%$ ). Mp: $151-153^{\circ} \mathrm{C}$. ESI-QToF MS $m / z$ $254.11655[\mathrm{M}]^{+}$. FTIR: $v_{\max }\left(\mathrm{cm}^{-1}\right), 3250(\mathrm{NH}), 1610(\mathrm{C}=\mathrm{O})$. ${ }^{1} \mathrm{H}-\mathrm{NMR}\left(500 \mathrm{MHz}, \mathrm{CDCl}_{3}\right) \delta 6.55(d, 1 \mathrm{H}, J=16 \mathrm{~Hz}), 7.75$ $(d, 1 \mathrm{H}, J=16 \mathrm{~Hz}), 7.54(d d, 2 \mathrm{H}, J=8.4 \mathrm{~Hz}), 7.37(d t$, $3 \mathrm{H}, J=2.6 \mathrm{~Hz}, J=8.4 \mathrm{~Hz}), 7.52(d d, 2 \mathrm{H}, J=8.4 \mathrm{~Hz})$, $6.87(d d, 2 \mathrm{H}, J=8.4 \mathrm{~Hz}), 3.85(\mathrm{~s}, 3 \mathrm{H}),{ }^{13} \mathrm{C}-\mathrm{NMR}(125 \mathrm{MHz}$, $\left.\mathrm{CDCl}_{3}\right) \delta 165.1,120.9,141.0,139.1,127.6,128.7,129.6$, $128.7,127.6,138.3,121.5,113.7,156.7,113.7,121.5$, 55.01 .

Nitration of cinnamic acid (2c) and nitration of cinnamamide compound (series 4)

Cinnamamide/cinnamic acid compound (4.05 mmol) was added to a mixture of $\mathrm{H}_{2} \mathrm{SO}_{4}(8.10 \mathrm{mmol})$ and $\mathrm{HNO}_{3}(8.10$ $\mathrm{mmol})$. The mixture was stirred in an oil bath at $50^{\circ} \mathrm{C}$, and the progress of the reaction was monitored by TLC. At the end of the reaction, the resulting mixture was diluted with ethyl acetate/water. The filtrate was washed with water (2 $\times 10 \mathrm{~mL}$ ) and dried with $\mathrm{Na}_{2} \mathrm{SO}_{4}$. The product was purified by column chromatography with the use of ethyl acetatehexane (2:1) as the eluent (Hurth et al. 2015; Lu et al. 2013; Montalbetti \& Falque 2005; Oxley et al. 2008; Rodrigues et al. 1999).

\section{(E)3-(4-nitrophenyl)cinnamic acid (2c)}

Yield: (1.51 g, 45\%). Mp: $147-148^{\circ} \mathrm{C}$. ESI-QToF MS $m / z$ 193.04678 $[\mathrm{M}+\mathrm{H}]^{+}$. FTIR: $v_{\max }\left(\mathrm{cm}^{-1}\right), 3039(\mathrm{NH}), 1687$ $(\mathrm{C}=\mathrm{O}), 1396(\mathrm{NO}) .{ }^{1} \mathrm{H}-\mathrm{NMR}\left(500 \mathrm{MHz}, \mathrm{CDCl}_{3} \delta 6.55(d\right.$, $1 \mathrm{H}, J=16 \mathrm{~Hz}), 7.75(d, 1 \mathrm{H}, J=16 \mathrm{~Hz}), 7.54(d, 2 \mathrm{H}, J=$ $8.4 \mathrm{~Hz}), 7.37(d, 2 \mathrm{H}, J=8.4 \mathrm{~Hz}),{ }^{13} \mathrm{C}-\mathrm{NMR}(125 \mathrm{MHz}$, $\left.\mathrm{CDCl}_{3}\right) \delta 172.8,117.4,147.2,134.1,128.5,129.1,130.8$, 129.1, 128.5.

\section{(E)3-(4-nitrophenyl) cinnamamide (4a)}

Yield: (0.392 g, 50.4\%). Mp: $147-148^{\circ} \mathrm{C}$. ESI-QToF MS $m / z 193.05678[\mathrm{M}+\mathrm{H}]^{+}$. FTIR: $v_{\max }\left(\mathrm{cm}^{-1}\right), 3039(\mathrm{NH}), 1687$
$(\mathrm{C}=\mathrm{O}), 1396$ (NO). ${ }^{1} \mathrm{H}-\mathrm{NMR}\left(500 \mathrm{MHz}, \mathrm{CDCl}_{3}\right) \delta 6.55(d$, $1 \mathrm{H}, J=16 \mathrm{~Hz}), 7.75(d, 1 \mathrm{H}, J=16 \mathrm{~Hz}), 7.54(d, 2 \mathrm{H}, J=$ $8.4 \mathrm{~Hz}), 7.37(d, 2 \mathrm{H}, J=8.4 \mathrm{~Hz}),{ }^{13} \mathrm{C}-\mathrm{NMR}(125 \mathrm{MHz}$, $\left.\mathrm{CDCl}_{3}\right) \delta 168.3,119.2,143.1,134.5,128.1,128.9,130.2$, $128.9,128.1$.

\section{(E)N-ethyl-(4-nitrophenyl)cinnamamide (4b)}

Yield: (0.490 g, 55\%). Mp: $138-139^{\circ} \mathrm{C}$. ESI-QToF MS m/z $221.10199[\mathrm{M}+\mathrm{H}]^{+}$, FTIR: $v_{\max }\left(\mathrm{cm}^{-1}\right), 3300(\mathrm{NH}), 1625$ $(\mathrm{C}=\mathrm{O}), 1418(\mathrm{NO}),{ }^{1} \mathrm{H}-\mathrm{NMR}\left(500 \mathrm{MHz}, \mathrm{CDCl}_{3}\right) \delta 6.55(d$, $1 \mathrm{H}, J=16 \mathrm{~Hz}), 7.75(d, 1 \mathrm{H}, J=16 \mathrm{~Hz}), 7.54(d, 2 \mathrm{H}, J=$ $8.4 \mathrm{~Hz}), 7.37(d, 2 \mathrm{H}, J=8.4 \mathrm{~Hz}), 3.42(m, 2 \mathrm{H}, J=2.6$ $\mathrm{Hz}), 1.20(t, 1 \mathrm{H}, J=7.15 \mathrm{~Hz}),{ }^{13} \mathrm{C}-\mathrm{NMR}\left(125 \mathrm{MHz}, \mathrm{CDCl}_{3}\right)$ $\delta 166.4,121.3,140.5,135.0,129.6,128.8,148.3,129.6$, $128.8,34.7,14.9$.

\section{(E)3-(4-nitrophenyl) N-propylcinnamamide (4c)}

Yield: (0.62 g, 65.4\%). Mp: 95-96 ${ }^{\circ} \mathrm{C}$. ESI-QToF MS $\mathrm{m} / \mathrm{z}$ $235.10663[\mathrm{M}+\mathrm{H}]^{+}$. FTIR: $v_{\max }\left(\mathrm{cm}^{-1}\right), 3275(\mathrm{NH}), 1631$ $(\mathrm{C}=\mathrm{O}), 1336(\mathrm{NO}) .{ }^{1} \mathrm{H}-\mathrm{NMR}\left(500 \mathrm{MHz}, \mathrm{CDCl}_{3}\right) \delta 6.48(d$, $1 \mathrm{H}, J=16 \mathrm{~Hz}), 7.60(d, 1 \mathrm{H}, J=16 \mathrm{~Hz}), 7.45(d, 2 \mathrm{H}, J=$ $7.8 \mathrm{~Hz}), 7.30(d, 2 \mathrm{H}, J=7.8 \mathrm{~Hz}), 3.34(q, 2 \mathrm{H}, J=6.5 \mathrm{~Hz})$, $1.58(m, 2 \mathrm{H}, J=7.15 \mathrm{~Hz}), 0.93(t, 3 \mathrm{H}, J=7.75 \mathrm{~Hz})$, ${ }^{13} \mathrm{C}-\mathrm{NMR}\left(125 \mathrm{MHz}, \mathrm{CDCl}_{3}\right) \delta 165.2,124.9,135.9,133.5$, $129.8,129.2,148.3,129.2,129.8,41.7,22.9,11.5$.

(E)3-(4-nitrophenyl) N-isopropylcinnamamide (4d) Yield: (0.544 g, 57.4\%). Mp: $154-155^{\circ} \mathrm{C}$. ESI-QTOF MS $m / z 235.10686[\mathrm{M}+\mathrm{H}]^{+}$, FTIR: $v_{\max }\left(\mathrm{cm}^{-1}\right), 3288(\mathrm{NH}), 1604$ $(\mathrm{C}=\mathrm{O}), 1344$ (NO). ${ }^{1} \mathrm{H}-\mathrm{NMR}\left(500 \mathrm{MHz}, \mathrm{CDCl}_{3}\right) \delta 6.46(d$, $1 \mathrm{H}, J=15.5 \mathrm{~Hz}), 7.67(d, 1 \mathrm{H}, J=15.5 \mathrm{~Hz}), 7.62(d, 2 \mathrm{H}$, $J=7.75 \mathrm{~Hz}), 8.23(d, 2 \mathrm{H}, J=7.75 \mathrm{~Hz}), 4.22(m, 1 \mathrm{H}, J$ $=6.25 \mathrm{~Hz}), 1.23(d, 6 \mathrm{H}, \mathrm{J}=6.75 \mathrm{~Hz}),{ }^{13} \mathrm{C}-\mathrm{NMR}(125 \mathrm{MHz}$, $\left.\mathrm{CDCl}_{3}\right) \delta 165.3,121.2,140.8,134.9,128.2,128.8,129.6$, 128.8, 128.2, 41.7, 22.9, 22.9.

\section{(E)3-(4-nitrophenyl) N-octylcinnamamide (4e)}

Yield: (0.6 g, 48.8\%). Mp: $84-85^{\circ} \mathrm{C}$. ESI-QToF MS $\mathrm{m} / \mathrm{z}$ $305.1897[\mathrm{M}+\mathrm{H}]^{+}$. FTIR: $v_{\max }\left(\mathrm{cm}^{-1}\right), 3300(\mathrm{NH}), 1625$ $(\mathrm{C}=\mathrm{O}), 1336(\mathrm{NO}) .{ }^{1} \mathrm{H}-\mathrm{NMR}\left(500 \mathrm{MHz}, \mathrm{CDCl}_{3}\right) \delta 6.46(d$, $1 \mathrm{H}, J=16 \mathrm{~Hz}), 7.64(d, 1 \mathrm{H}, J=16 \mathrm{~Hz}), 7.48(d, 2 \mathrm{H}, J$ $=7.8 \mathrm{~Hz}), 7.32(d, 2 \mathrm{H}, J=7.8 \mathrm{~Hz}), 3.37(q, 2 \mathrm{H}, J=6.5$ $\mathrm{Hz}), 1.24(m, 10 \mathrm{H}, J=7.8 \mathrm{~Hz}), 1.56(m, 2 \mathrm{H}, J=7.8 \mathrm{~Hz})$, $0.85(t, 3 \mathrm{H}, J=6.5 \mathrm{~Hz}),{ }^{13} \mathrm{C}-\mathrm{NMR}\left(125 \mathrm{MHz}, \mathrm{CDCl}_{3}\right) \delta$ $164.9,124.9,135.8,133.4,129.8,129.2,148.1,129.2$, $129.8,40.04,31.9,27.1,29.3,29.3,29.7,22.7,14.2$.

Biological activity assays

Biological activity assays for the $\alpha$-glucosidase enzyme inhibitory activity has been reported by Kim et al. (2004). $S$. cerevisiae $\alpha$-glucosidase $(0.5 \mathrm{mg})$ was dissolved in 5 $\mathrm{mL}$ phosphate buffer at $\mathrm{pH} 7$, and $100 \mathrm{mg}$ bovine was dissolved in $5 \mathrm{~mL}$ phosphate buffer. Both enzyme and bovine solutions were mixed into $40 \mathrm{~mL}$ phosphate buffer at $\mathrm{pH} 7$. Into a test tube was added a solution of $250 \mu \mathrm{L}$ of $20 \mathrm{mM}$ p-NPG, $495 \mu \mathrm{L} 100 \mathrm{mM}$ phosphate buffer, and 5 $\mu \mathrm{L}$ sample solution of each compound synthesized and incubated for $5 \mathrm{~min}$ at $37^{\circ} \mathrm{C}$. Next, $250 \mu \mathrm{L}$ of the enzyme was added and incubated for $15 \mathrm{~min}$ at $37^{\circ} \mathrm{C}$. The reaction 
was stopped by adding $1000 \mu \mathrm{L}$ of $\mathrm{Na}_{2} \mathrm{CO}_{3}$ solution. Absorbance was then measured using a spectrophotometer at a wavelength of $400 \mathrm{~nm}$. Percentage enzyme inhibition was measured as ((absorbance control-absorbance test)/ absorbance control) $\times 100$.

Kim et al. (2004) was reported biological activity assays for the $\alpha$-glucosidase enzyme which have been modified. Rat intestinal acetone powder $\alpha$-glucosidase (500 $\mathrm{mg}$ ) was homogenized in $10 \mathrm{~mL}$ of $0.9 \% \mathrm{NaCl}$ solution. After centrifugation (5000 rpm for $30 \mathrm{~min}$ ), the solution was used in the experiment. Into a test tube was added a solution of $250 \mu \mathrm{L}$ of $20 \mathrm{mM}$-NPG, $595 \mu \mathrm{L} 100 \mathrm{mM}$ phosphate buffer, and $5 \mu \mathrm{L}$ sample solution. After incubation for $5 \mathrm{~min}$ at $37^{\circ} \mathrm{C}, 150 \mu \mathrm{L}$ of the enzyme was added and again incubated for $15 \mathrm{~min}$ at $37^{\circ} \mathrm{C}$. The reaction was stopped by the addition of $1000 \mu \mathrm{L}$ of $\mathrm{Na}_{2} \mathrm{CO}_{3}$ solution. Absorbance of the solution was then measured using a spectrophotometer at a wavelength of $400 \mathrm{~nm}$. Percentage enzyme inhibition was measured as ((absorbance controlabsorbance test)/absorbance control $) \times 100$.

\section{Measurement of kinetic constant}

In order to investigate the type of inhibition, $\alpha$-glucosidase enzyme kinetics analysis was performed according to a reported method (Adisakwattana et al. 2009). Kinetic analyses of the samples were performed using two reaction systems, i.e. the reaction system without the inhibitor and the reaction system with the sample inhibitor. The samples tested for their inhibitory kinetics were the best samples from previous inhibition test results (biological activity assay 2.2.4). The test was performed using different substrate concentrations ( $\mathrm{p}$-NPG), i.e. $0.5,1,35,10 \mu \mathrm{M}$ in phosphate buffer $\mathrm{pH} 7$. The reaction system mixture was similar to the mixture used for evaluating $\alpha$-glucosidase inhibition. The absorbance was read at $400 \mathrm{~nm}$, and the experiment was performed with 3 replicates. The type of inhibition was calculated on the basis of a LineweaverBurk plot using reciprocally plotted data (substrate concentration on the horizontal axis and velocity on the vertical axis).

\section{RESULTS AND DISCUSSION}

\section{SYNTHESIS OF CINNAMAMIDE DERIVATIVES}

Synthetic cinnamamide derivatives were synthesized and assayed for their $\alpha$-glucosidase inhibitory activities (Scheme 1 and Table 1). Basic hydrolysis of methyl transcinnamate 1 produced cinnamic acid 2. Treatment of cinnamic acid 2 with $\mathrm{SOCl}_{2}$ and alkylamine afforded the corresponding cinnamamide derivatives (3a-3g) in good yields. Thus obtained cinnamamide derivatives ( $3 \mathrm{a}-3 \mathrm{~g})$ were then nitrated with sulfuric acid and nitric acid, and nitro cinnamamide derivatives $(4 a-4 e)$ were obtained in moderate yields. The structures of cinnamamide derivatives (3a-3g) and (4a-4e) were characterized by UV, IR, melting point analysis, ${ }^{1} \mathrm{H}-\mathrm{NMR},{ }^{13} \mathrm{C}-\mathrm{NMR}$, GCMS, LCMS, and ESIQTOF mass spectral analysis. The UV absorption spectra<smiles>[R]C(=O)/C=C/c1ccc([R])cc1</smiles>

TABLE 1. Synthesis of cinnamamide derivatives

\begin{tabular}{|c|c|c|c|c|c|}
\hline Entry & Sample & $\mathrm{R}$ & $\mathrm{R}^{\prime}$ & Melting point $\left({ }^{\circ} \mathrm{C}\right)$ & Yield (\%) \\
\hline 1 & Methyl cinnamate $\mathbf{1}$ & $\mathrm{OCH}_{3}$ & $\mathrm{H}$ & $34-35$ & 65 \\
\hline 2 & Cinnamic acid $\mathbf{2}$ & $\mathrm{OH}$ & $\mathrm{H}$ & $133-134$ & 85.5 \\
\hline 3 & Cinnamamide 3a & $\mathrm{NH}_{2}$ & $\mathrm{H}$ & $145-148$ & 80 \\
\hline 4 & Ethylcinnamamide $\mathbf{3 b}$ & $\mathrm{NHCH}_{2} \mathrm{CH}_{3}$ & $\mathrm{H}$ & $85-87$ & 75 \\
\hline 5 & Propylcinnamamide $\mathbf{3 c}$ & $\mathrm{NH} \mathrm{CH}_{2} \mathrm{CH}_{2} \mathrm{CH}_{3}$ & $\mathrm{H}$ & $74-75$ & 82 \\
\hline 6 & Isopropylcinnamamide $\mathbf{3 d}$ & $\mathrm{NHCH}\left(\mathrm{CH}_{3}\right)_{2}$ & $\mathrm{H}$ & $95-97$ & 78 \\
\hline 7 & Octylcinnamamide $\mathbf{3 e}$ & $\mathrm{NH}\left(\mathrm{CH}_{2}\right)_{7} \mathrm{CH}_{3}$ & $\mathrm{H}$ & $74-75$ & 82.4 \\
\hline 8 & Phenylcinnamamide $\mathbf{3 f}$ & $\mathrm{NHC}_{6} \mathrm{H}_{5}$ & $\mathrm{H}$ & $77-79$ & 78 \\
\hline 9 & p-Methoxyphenylcinnamamide $\mathbf{3 g}$ & $\mathrm{NHC}_{6} \mathrm{H}_{4} \mathrm{OCH}_{3}$ & $\mathrm{H}$ & $151-153$ & 78 \\
\hline 10 & (E)3-(4-nitrophenyl)acrylic acid $\mathbf{2 a}$ & $\mathrm{OH}$ & $\mathrm{NO}_{2}$ & $165-168$ & 56.4 \\
\hline 11 & (E)3-(4-nitrophenyl)acrylamide $\mathbf{4 a}$ & $\mathrm{NH}_{2}$ & $\mathrm{NO}_{2}$ & $147-148$ & 50.4 \\
\hline 12 & (E) N-ethyl-(4-nitrophenyl) acrylamide $\mathbf{4 b}$ & $\mathrm{NHCH}_{2} \mathrm{CH}_{3}$ & $\mathrm{NO}_{2}$ & $138-139$ & 55 \\
\hline 13 & (E)3-(4-nitrophenyl)propylacrylamide $\mathbf{4 c}$ & $\mathrm{NH} \mathrm{CH}_{2} \mathrm{CH}_{2} \mathrm{CH}_{3}$ & $\mathrm{NO}_{2}$ & $95-96$ & 65.4 \\
\hline 14 & (E)3-(4-nitrophenyl) $N$-isopropylacrylamide4d & $\mathrm{NHCH}\left(\mathrm{CH}_{3}\right)_{2}$ & $\mathrm{NO}_{2}$ & $154-155$ & 57.4 \\
\hline 15 & (E)3-(4-nitrophenyl) $\mathrm{N}$-octylacrylamide $4 \mathbf{e}$ & $\mathrm{NH}\left(\mathrm{CH}_{2}\right)_{7} \mathrm{CH}_{3}$ & $\mathrm{NO}_{2}$ & $84-85$ & 48.8 \\
\hline
\end{tabular}




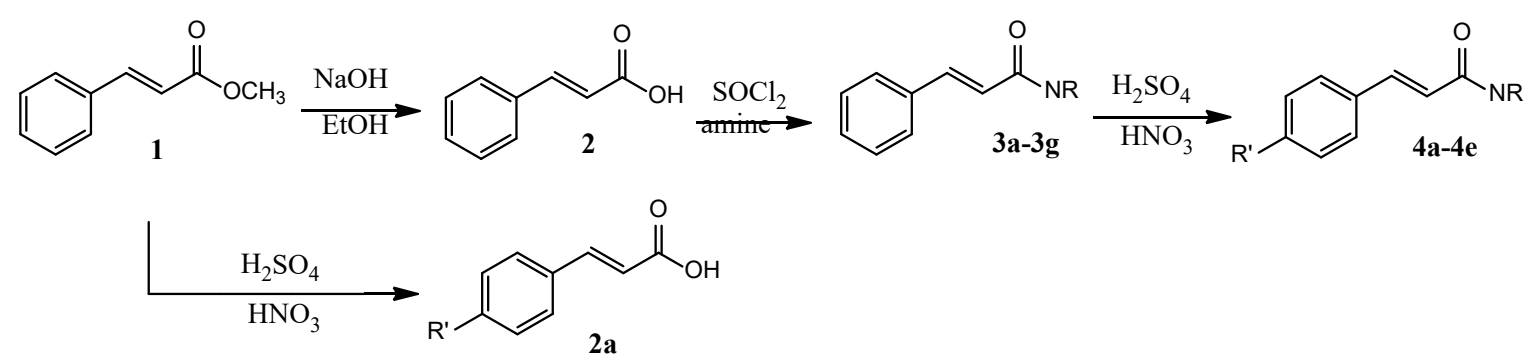

SCHEME 1. Synthesis of cinnamamide derivatives

of $3 a-3 g$ and $4 a-4 e$ exhibited their main peak at $280 \mathrm{~nm}$, upon introduction of the carbonyl and amide group, respectively. The IR spectrum of $3 \mathrm{a}-3 \mathrm{~g}$ showed an absorption peak at $3250-3369 \mathrm{~cm}^{-1}$ due to the amine (-NH) stretching, and at $1662-1656 \mathrm{~cm}^{-1}$ due to the carbonyl amide $(-\mathrm{N}-\mathrm{C}=\mathrm{O})$ stretching. The IR spectrum of $4 \mathrm{a}-4 \mathrm{e}$ showed an absorption peak at $3039-3300 \mathrm{~cm}^{-1}$ due to the amine (-NH) stretching, at $1604-1687 \mathrm{~cm}^{-1}$ due to carbonyl amide $(-\mathrm{N}-\mathrm{C}=\mathrm{O})$ stretching, and at $1336-1418 \mathrm{~cm}^{-1}$ due to the nitro $\left(-\mathrm{NO}_{2}\right)$ stretching. The ${ }^{1} \mathrm{H}$ NMR spectrum of $3 \mathrm{a}-3 \mathrm{~g}$ and $4 \mathrm{a}-4 \mathrm{e}$ showed a cinnamic acid backbone through doublet $(d)$ at $\delta 6.43 \mathrm{ppm}$, doublet at $\delta 7.64 \mathrm{ppm}$, doubletdoublet $(d d)$ at $\delta 7.47 \mathrm{ppm}$, and doublet-triplet $(d t)$ at $\delta$ $7.35 \mathrm{ppm}$, while an alkyl amine was evident by the following peak characteristics: ethylamide: triplets at $\delta$ $1.20 \mathrm{ppm}$, quartet at $\delta 3.42 \mathrm{ppm}$; propylamide: quartet at $\delta 3.34 \mathrm{ppm}$, multiplets at $\delta 1.58 \mathrm{ppm}$, triplets at $\delta 0.93$ ppm; isopropylamide: multiplet at $\delta 4.22 \mathrm{ppm}$, doublets at $\delta 1.22$ ppm; octylamide: quartet at $\delta 3.37 \mathrm{ppm}$, multiplets at $\delta 1.24 \mathrm{ppm}$, multiplets at $\delta 1.56 \mathrm{ppm}$, and triplets at $\delta$ $0.85 \mathrm{ppm}$. The ${ }^{13} \mathrm{C}$ NMR spectrum of $3 \mathrm{a}-3 \mathrm{~g}$ and $4 \mathrm{a}-4 \mathrm{e}$ showed a cinnamic acid backbone through signals at $\delta$ $119.2,128.1,128.9,130.2,130.5,134.5,143.1$, and 168.3 ppm, while an alkyl amine was evident by the following signals: ethylamide: 34.7 and 14.8 ppm; propylamide: 41.6 , 22.9, and $11.6 \mathrm{ppm}$; isopropylamide: 41.7 and $22.9 \mathrm{ppm}$; octylamide: $40.0,31.8,29.7,29.4,29.3,27.1,22.7$, and
14.2 ppm. Compounds 1, 2, 3a-3g, and 4a-4e were also identified by mass spectral analysis.

\section{BIOLOGICAL ACTIVITY}

$\alpha$-Glucosidase inhibitory activity

Glucosidase is responsible for the catalytic disruption of glycosidic bonds, which depends explicitly on the amount of monosaccharides, the position of the cleavage site, and the configuration of the hydroxyl group in the substrate. Herein, we have synthesized 13 derivatives and report their inhibitory effects on $\alpha$-glucosidase from $S$. cerevisiae and rat intestinal tissue. Ten compounds showed a more potent inhibition than standard acarbose, and five compounds showed a more potent inhibition than the standard 1-deoxynojirimycin. However, all cinnamamide derivatives showed better inhibition of $\alpha$-glucosidase than the starting material.

Thirteen compounds showed $\alpha$-glucosidase activity with $\mathrm{IC}_{50}$ values of $0.71 \pm 0.492-4.02 \pm 0.025 \mathrm{mM}$ (1-deoxynojirimycin: $0.97 \pm 0.019 \mathrm{mM}$ ) and acarbose $\left(\mathrm{IC}_{50}\right.$ $=1.78 \pm 0.727 \mathrm{mM})$. These compounds, based on the cinnamamide skeleton, only slightly differed from each other in their $\alpha$-glucosidase inhibitory potential possible because of the different lengths of alkyl amine chain. Propylcinnamamide $3 \mathrm{c}\left(\mathrm{IC}_{50}=0.71 \pm 0.492 \mathrm{mM}\right)$, nitro propylcinnamamide $4 \mathrm{c}\left(\mathrm{IC}_{50}=0.75 \pm 0.066 \mathrm{mM}\right)$,

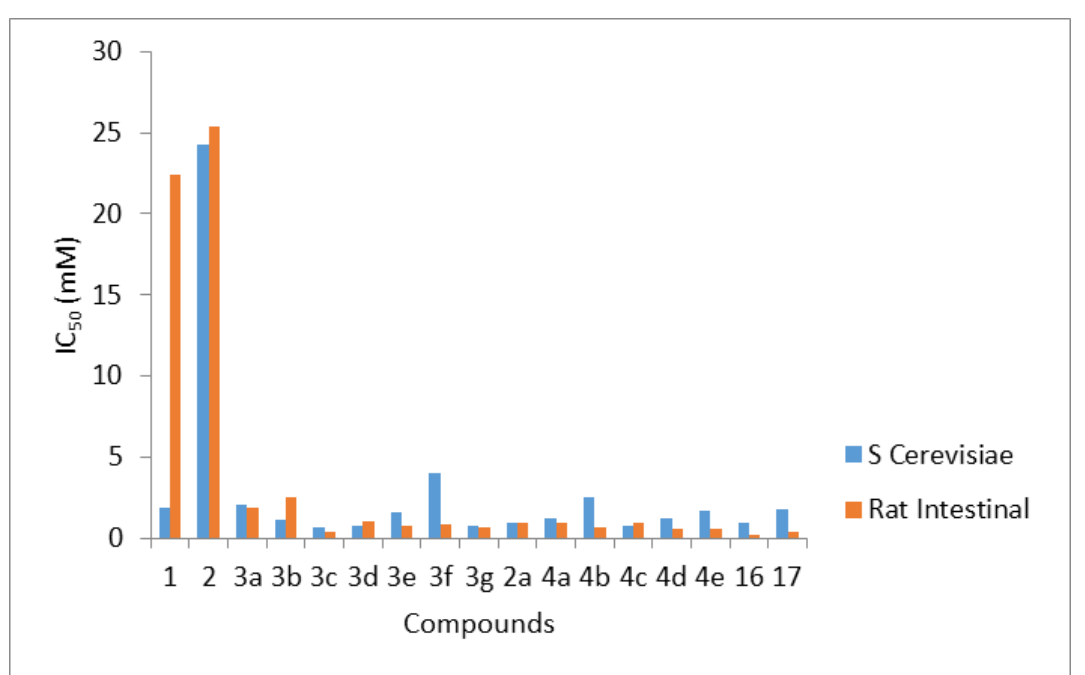

FIGURE 1. IC50 values of cinnamamide derivatives for inhibition of $\alpha$-glucosidase 
<smiles>[R]C(=O)/C=C/c1ccc([R])cc1</smiles>

TABLE 2. IC50 values of cinnamamide derivatives for inhibition of $\alpha$-glucosidase

\begin{tabular}{|c|c|c|c|c|c|}
\hline \multirow{2}{*}{ Entry } & \multirow{2}{*}{ Sample } & \multirow{2}{*}{$\mathrm{R}$} & \multirow{2}{*}{$\mathrm{R}^{\prime}$} & \multicolumn{2}{|c|}{$\mathrm{IC}_{50} \pm \mathrm{SEM}^{*}(\mathrm{mM})$} \\
\hline & & & & S. cerevisiae & Rat intestinal \\
\hline 1 & Methyl cinnamate $\mathbf{1}$ & $\mathrm{OCH}_{3}$ & $\mathrm{H}$ & $1.88 \pm 0.022$ & $22.42 \pm 0.011$ \\
\hline 2 & Cinnamic acid 2 & $\mathrm{OH}$ & $\mathrm{H}$ & $24.28 \pm 0.019$ & $25.37 \pm 0.011$ \\
\hline 3 & Cinnamamide 3a & $\mathrm{NH}_{2}$ & $\mathrm{H}$ & $2.05 \pm 0.020$ & $1.86 \pm 0.047$ \\
\hline 4 & Ethylcinnamamide $\mathbf{3 b}$ & $\mathrm{NHCH}_{2} \mathrm{CH}_{3}$ & $\mathrm{H}$ & $1.15 \pm 1.006$ & $2.52 \pm 0.253$ \\
\hline 5 & Propylcinnamamide $\mathbf{3 c}$ & $\mathrm{NH} \mathrm{CH}_{2} \mathrm{CH}_{2} \mathrm{CH}_{3}$ & $\mathrm{H}$ & $0.71 \pm 0.492$ & $0.37 \pm 0.145$ \\
\hline 6 & Isopropylcinnamamide $\mathbf{3 d}$ & $\mathrm{NHCH}\left(\mathrm{CH}_{3}\right)_{2}$ & $\mathrm{H}$ & $0.81 \pm 0.757$ & $1.04 \pm 0.054$ \\
\hline 7 & Octylcinnamamide $\mathbf{3 e}$ & $\mathrm{NH}\left(\mathrm{CH}_{2}\right)_{7} \mathrm{CH}_{3}$ & $\mathrm{H}$ & $1.59 \pm 0.008$ & $0.80 \pm 0.092$ \\
\hline 8 & Phenylcinnamamide $\mathbf{3 f}$ & $\mathrm{NHC}_{6} \mathrm{H}_{5}$ & $\mathrm{H}$ & $4.02 \pm 0.025$ & $0.86 \pm 0.184$ \\
\hline 9 & p-Methoxyphenylcinnamamide $\mathbf{3 g}$ & $\mathrm{NHC}_{6} \mathrm{H}_{4} \mathrm{OCH}_{3}$ & $\mathrm{H}$ & $0.80 \pm 1.416$ & $0.71 \pm 0.160$ \\
\hline 10 & (E)3-(4-nitrophenyl)acrylic acid 2a & $\mathrm{OH}$ & $\mathrm{NO}_{2}$ & $0.95 \pm 0.008$ & $0.99 \pm 0.239$ \\
\hline 11 & (E)3-(4-nitrophenyl)acrylamide $\mathbf{4 a}$ & $\mathrm{NH}_{2}$ & $\mathrm{NO}_{2}$ & $1.26 \pm 0.047$ & $0.96 \pm 0.044$ \\
\hline 12 & (E)N-ethyl-(4-nitrophenyl)acrylamide $\mathbf{4 b}$ & $\mathrm{NHCH}_{2} \mathrm{CH}_{3}$ & $\mathrm{NO}_{2}$ & $2.59 \pm 0.052$ & $0.66 \pm 0.055$ \\
\hline 13 & (E)3-(4-nitrophenyl) N-propylacrylamide $\mathbf{4 c}$ & $\mathrm{NH} \mathrm{CH}_{2} \mathrm{CH}_{2} \mathrm{CH}_{3}$ & $\mathrm{NO}_{2}$ & $0.75 \pm 0.066$ & $0.98 \pm 0.217$ \\
\hline 14 & (E)3-(4-nitrophenyl)N-isopropylacrylamide $\mathbf{4 d}$ & $\mathrm{NHCH}\left(\mathrm{CH}_{3}\right)_{2}$ & $\mathrm{NO}_{2}$ & $1.21 \pm 0.094$ & $0.59 \pm 0.418$ \\
\hline 15 & (E)3-(4-nitrophenyl) N-octylacrylamide $4 \mathbf{e}$ & $\mathrm{NH}\left(\mathrm{CH}_{2}\right)_{7} \mathrm{CH}_{3}$ & $\mathrm{NO}_{2}$ & $1.73 \pm 0.074$ & $0.59 \pm 0.017$ \\
\hline 16 & 1-Deoxynojirimycin & & & $0.97 \pm 0.019$ & $0.20 \pm 0.047$ \\
\hline 17 & Acarbose & & & $1.78 \pm 0.727$ & $0.46 \pm 0.251$ \\
\hline
\end{tabular}

SEM, standard error of mean; 1-deoxynojirimycin and acarbose were used as standards

methoxyphenylcinnamamide $3 \mathrm{~g}\left(\mathrm{IC}_{50}=0.80 \pm 1.416\right)$, isopropylcinnamamide $3 \mathrm{~d}\left(\mathrm{IC}_{50}=0.81 \pm 0.757 \mathrm{mM}\right)$, and nitro cinnamic acid $2 \mathrm{a}\left(\mathrm{IC}_{50}=0.95 \pm 0.008 \mathrm{mM}\right)$ showed the best activities, which were better than those of the standard 1-deoxynojirimycin and acarbose (Table 2).

In the assay for inhibitory potential against rat intestinal $\alpha$-glucosidase, compounds $3 \mathrm{c}, 3 \mathrm{~g}, 4 \mathrm{~b}, 4 \mathrm{~d}$, and $4 \mathrm{e}\left(\mathrm{IC}_{50}=0.37 \pm 0.145 \mathrm{mM}, 0.71 \pm 0.160 \mathrm{mM}, 0.66 \pm 0.055\right.$ $\mathrm{mM}, 0.59 \pm 0.418 \mathrm{mM}$, and $0.59 \pm 0.017 \mathrm{mM}$, respectively) had potent activities, which were equivalent to those of acarbose and 1-deoxynojirimycin $\left(\mathrm{IC}_{50}=0.46 \pm 0.251 \mathrm{mM}\right.$ and $0.20 \pm 0.047 \mathrm{mM}$, respectively). Methyl transcinnamate (1) and trans-cinnamic acid (2) were found to be inactive ( $\left.\mathrm{IC}_{50}>20 \mathrm{mM}\right)$. Propylcinnamamide (3c) had very potent inhibitory activity $\left(\mathrm{IC}_{50}=0.37 \pm 0.145 \mathrm{mM}\right)$ and was the most active among all cinnamamide derivatives. These results suggested that presence of the amide group at the carboxyl group of cinnamic acid moiety is necessary to increase $\alpha$-glucosidase inhibitory activity. Increasing the chain length of alkylamine increased $\alpha$-glucosidase inhibitory activity up to an optimum chain length, any increase beyond this length decreased the inhibitory activity; Propylcinnamamide had an optimum chain length of alkyl amine, and therefore, had the best activity on intestinal $\alpha$-glucosidase. Isopropylcinamamide showed lesser activity than propylcinnamamide because its alkyl amine was bulkier.

Figure 1 shows that methyl trans-cinnamate and transcinnamic acid had a weak inhibitory activity against $S$. cerevisiae and rat intestinal $\alpha$-glucosidase. Cinnamamide derivatives $3 \mathrm{a}-4 \mathrm{e}$ were more potent than methyl transcinnamate and trans-cinnamic acid. Propylcinnamamide showed the best activity on intestinal $\alpha$-glucosidase inhibition among the cinnamamide derivatives and was more potent than acarbose.

Inhibition kinetics of propylcinnamamide on $\alpha$-glucosidase The Lineweaver-Burk plot of $\alpha$-glucosidase inhibition kinetics is shown in Figure 2 and shows that the mechanism of $\alpha$-glucosidase inhibition of propylcinnamamide (5) is competitive. The mechanism of inhibition of propylcinamide against the $\alpha$-glucosidase enzyme in this study was studied through the LineweaverBurk curve based on the reaction of enzymes with substrates and enzymes with substrates and inhibitors. Based on the results of the Lineweaver-burk plot, the 


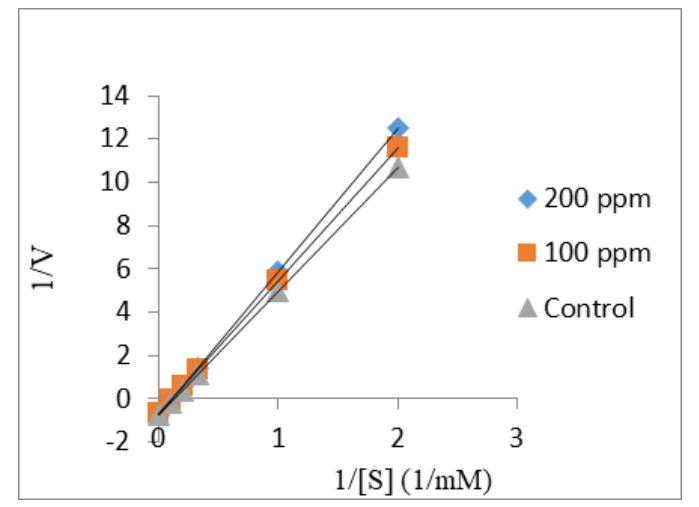

(a)

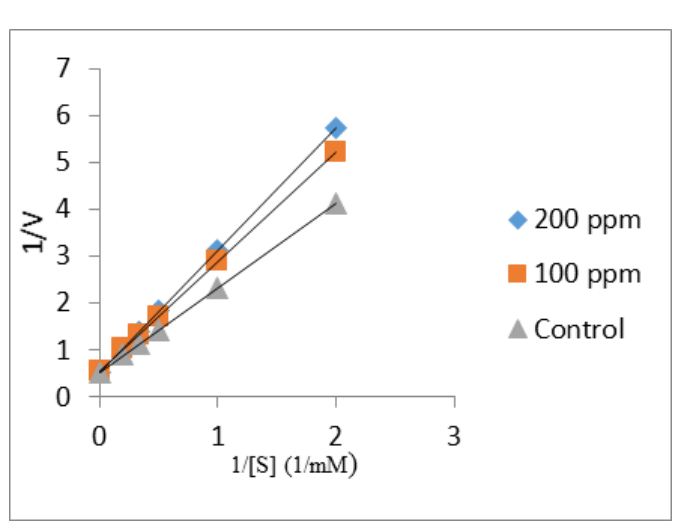

(b)

FIGURE 2. Lineweaver-Burk plot for inhibitory activity of propylcinnamamide against

(a) S. cerevisiae $\alpha$-glucosidase and (b) rat intestinal $\alpha$-glucosidase

propylsamamide compound works competitively (Figure 2), when $1 /[\mathrm{S}]$ approaches 0 , the maximum reaction speed (V max) is not affected by the presence of an inhibitor. Therefore, when the substrate concentration is high, $\mathrm{V}$ max in systems with inhibitors is equal to or close to $\mathrm{V}$ max with systems without inhibitors. Inhibitors that work competitively do not affect the value of Vmax, but increase the value of Km (Murray et al. 2003).

\section{CONCLUSION}

Thirteen compounds showed $\alpha$-glucosidase activity $(S$. cerevisiae) with $\mathrm{IC}_{50}$ values of 0.71-4.02 $\mathrm{mM}$. Propylcinnamamide $(3 \mathrm{c})\left(\mathrm{IC}_{50}=0.71 \pm 0.492 \mathrm{mM}\right)$, nitro propyl cinnamamide $(4 \mathrm{c})\left(\mathrm{IC}_{50}=0.75 \pm 0.066\right)$, methoxyphenylcinnamamide $(3 \mathrm{~g})\left(\mathrm{IC}_{50}=0.80 \pm 1.416\right)$, isopropylcinnamamide $(3 \mathrm{~d}))\left(\mathrm{IC}_{50}=0.81 \pm 0.757\right)$, and nitro cinnamic acid $(2 \mathrm{a})\left(\mathrm{IC}_{50}=0.95 \pm 0.008\right)$ showed the best activities, which were greater than those of the standards 1-deoxynojirimycin and acarbose. Compounds $5,9,12,14$, and $15\left(\mathrm{IC}_{50}=0.37 \mathrm{mM}, 0.71 \mathrm{mM}, 0.66 \mathrm{mM}\right.$, $0.59 \mathrm{mM}$, and $0.59 \mathrm{mM}$, respectively) showed potent $\alpha$-glucosidase inhibitory activity (rat intestinal). The presence of amine substituents on cinnamic acid was responsible for the increase in the $\alpha$-glucosidase inhibitory activity of the parent compound. An increase in the bulkiness and chain length of the amine substituents decreased the inhibitory activity. Propylcinnamamide (5) showed the highest inhibitory activity among all cinnamamide derivatives, and it acted via a competitive inhibitory mechanism. This study highlights compounds with the potential ability to regulate blood glucose levels and could be further developed for application in a clinical setting.

\section{ACKNOWLEDGEMENTS}

We would like to thank the Ministry of Research and Technology of Higher Education No.35/M/KP/IX/2018 for their support in providing funding.

\section{REFERENCES}

Adisakwattana, S., Charoenlertkul, P. \& Yibchok-anun, S. 2009. $\alpha$-Glucosisade inhibitory activity of cyaniding-3galactoside and synergitic effect with acarbose. Journal of Enzyme Inhibition and Medicinal Chemistry 24: 65-69.

Adisakwattana, S., Sookkongwaree, K., Roengsumran, S., Petsom, A., Ngamrojnavanich, N., Chavasiri, W. \& Yibchok-Anun, S.D. 2004. Structure activity relationships of trans-cinnamic acid derivatives on $\alpha$-glucosidase inhibition. Bioorg. Med. Chem. Lett. 14: 2893-2894.

Babu, P.S., Prabuseenivasan, S. \& Ignacimuthu, S. 2007. Cinnamaldehyde a potential antidiabetic agent. Phytomedicine 14: 15-20.

Bösenberg, L.H. \& van Zyl, D.G. 2008. The mechanism of action of oral antidiabetic drugs: A review of recent literature. Endocr. Metab. Diab. South Afr. 13(3): 80-88.

Carvalho, S.A., Silva, E.F., Souza, M.V.N., Lourenc, M.C.S. \& Vicente, F.R. 2008. Synthesis and antimycobacterial evaluation of new trans-cinnamic acid hydrazide derivatives. Bioorg. Med. Chem. Lett. 18: 538-544.

Davies, G. \& Henrissat, B. 1995. Structures and mechanisms of glycosyl hydrolases. Structure 3: 853-858.

Fischer, P.B., Collin, M., Karlsson, G.B., James, W., Butters, T.D., Davis, S.J. \& Platt, F.M. 1995. The $\alpha$-glucosidase inhibitor N-Butyldeoxynojirimycin inhibits human immunodeficiency virus entry at the level of post-CD4 binding. J. Virol. 69: 5791-5796.

Huang, D.W., Shen, S.C. \& Wu, J.S.B. 2009. Effects of caffeic acid and cinnamic acid on glucose uptake in insulinresistant mouse hepatocytes. J. Agric. Food Chem. 57: 7687-7692.

Hurth, K., Jacquier, S., Lehmann, H. \& Wilcken, R. 2015. Regioselective nitration of 3-fluoro-2-substituted benzoic acids. Tetrahedron Lett. 56: 2860-2860.

Kim, Y.M., Wang, M.H. \& Rhee, H.I. 2004. A novel alphaglucosidase inhibitor from pine bark. Carbohydr. Res. 339: 715-720.

Liu, Y., Zou, L., Ma, L., Chen, W.H., Wang, B. \& Xu, Z.L. 2006. Synthesis and pharmacologies activies xanthone derivatives as alpha glucosidase inhibitors. Bioorg. Med. Chem. 14: 5683-5688

Lu, Y., Li, Y., Zhang, R., Jin, K. \& Duan, C. 2013. Regioselective ortho-nitration of $N$-phenyl carboxamides and primary anilines using bismuth nitrate/acetic anhydride. Tetrahedron 69: 9422-9426. 
Matsuura, H., Miyazaki, H. \& Asakawa, C. 2004. Isolation of $\alpha$-glusosidase inhibitors from hyssop (Hyssopus officinalis). Phytochemistry 65: 91-95.

Mehta, A., Zitzmann, N., Rudd, P.M. \& Block, T.M. 1998 $\alpha$-Glucosidase inhibitors as potential broad based anti-viral agents. FEBS Lett. 430: 17-20.

Melo, E.B. \& Carvalho, I. 2006. $\alpha$ - and $\beta$-glucosidase inhibitors: Chemical structure and biological activity. Tetrahedron 62 10277-10280.

Mohammed, K., Rahim, F., Wadood, A., Kosar, N., Taha, M., Lalani, S. \& Perveen, S. 2014. Synthesis and molecular docking studies of potent $\alpha$-glucosidase inhibitors based on biscoumarin skeleton. Eur. J. Med. Chem. 81: 245-250.

Montalbetti, C.A. \& Falque, V. 2005. Amide bond formation and peptide coupling. Tetrahedron 61: 10827-10832.

Murray, K., Granner, D.K., Mayes, P. \& Rodwell, V. 2003. Harper's Illustrated Biochemistry. 26th ed. Longe Medical Pub.

Narender, T., Shweta, S., Tiwari, P., Reddy, K.P., Khaliq, T., Prathipati, P. \& Raj, K. 2007. Antihyperglycemic and antidyslipidemic agent from Aegle marmelos. Bioorg. Med. Chem. Lett. 17: 1808-1814.

Oxley, J.C., Smith, J.L., Moran, J.S., Canino, J.N. \& Almog, J. 2008. Aromatic nitration using nitroguanidine and EGDN. Tetrahedron Lett. 49: 4449-4454.

Park, J.E., So, H.P., Jung, Y.W., Hye, S.H., Jaeho, C. \& Heeseob, L. 2013. Enzymatic properties of a thermostable $\alpha$-glucosidase from acidothermophilic crenarchaeon Sulfolobus tokodaii Strain 7. J. Microbiol. Biotechnol. 23(1): 56-63.

Park, H., Hwang, K.Y., Oh, H., Kim, H., Yeon, J. \& Kim, K. 2008a. Discovery of novel $\alpha$-glucosidase inhibitors based on the virtual screening with the homology-modeled protein structure. Bioorg. Med. Chem. 16: 284-286.

Park, J., Ko, S. \& Park, H. 2008b. Toward the virtual screening of $\alpha$-glucosidase inhibitors with the homology-modeled protein structure. Bull. Korean Chem. Soc. 29: 921-925.
Phuwapraisirisan, P., Puksasook, T., Jong-aramruang, J. \& Kokpol, U. 2008. Phenylethyl cinnamides: A new series of $\alpha$ -glucosidase inhibitors from the leaves of Aegle marmelos. Bioorg. Med. Chem. Lett. 18: 4956-4960.

Pili, R., Chang, J.A., Partis, R.A., Mueller, R.J., Chrest, F. \& Passaniti, A. 1995. The alpha-glucosidase I inhibitor castanospermine alters endothelial cell glycosylation, prevents angiogenesis, and inhibits tumor growth. Cancer Res. 55: 2920-2926.

Rodrigues, J.A.R., de Oliveira Filho, A.P., Moran, P.J.S. \& Custódio, R. 1999. Regioselectivity of the nitration of phenol by acetyl nitrate adsorbed on silica gel. Tetrahedron. 55(22): 6733-6738.

Van de Laar, F.A. 2008. $\alpha$-Glucosidase inhibitors in the early treatment of Type 2 diabetes. Health Risk Manag. 4: 11891195 .

Teni Ernawati* \& Muhamad Hanafi

Research Center for Chemistry

Indonesian Institute of Sciences (LIPI)

Kawasan Puspiptek, Serpong Tangerang Selatan

Banten 15314

Indonesia

Abdul Mun'im \& Arry Yanuar

Faculty of Pharmacy

Universitas Indonesia

Depok 16242

Indonesia

*Corresponding author; email: teni.ernawati.lipi@gmail.com

Received: 5 September 2019

Accepted: 7 November 2019 